\title{
Modeling the Impact of Climate Change on the Dynamics of Rift Valley Fever
}

\author{
Saul C. Mpeshe, ${ }^{1}$ Livingstone S. Luboobi, ${ }^{1,2}$ and Yaw Nkansah-Gyekye ${ }^{1}$ \\ ${ }^{1}$ School of CoCSE, Nelson Mandela African Institution of Science and Technology, P.O. Box 447, Arusha, Tanzania \\ ${ }^{2}$ Department of Mathematics, Makerere University, P.O. Box 7062, Kampala, Uganda \\ Correspondence should be addressed to Saul C. Mpeshe; mpeshes@nm-aist.ac.tz
}

Received 30 August 2013; Revised 20 January 2014; Accepted 3 February 2014; Published 30 March 2014

Academic Editor: Gabriel Turinici

Copyright (c) 2014 Saul C. Mpeshe et al. This is an open access article distributed under the Creative Commons Attribution License, which permits unrestricted use, distribution, and reproduction in any medium, provided the original work is properly cited.

\begin{abstract}
A deterministic SEIR model of rift valley fever (RVF) with climate change parameters was considered to compute the basic reproduction number $\mathscr{R}_{0}$ and investigate the impact of temperature and precipitation on $\mathscr{R}_{0}$. To study the effect of model parameters to $\mathscr{R}_{0}$, sensitivity and elasticity analysis of $\mathscr{R}_{0}$ were performed. When temperature and precipitation effects are not considered, $\mathscr{R}_{0}$ is more sensitive to the expected number of infected Aedes spp. due to one infected livestock and more elastic to the expected number of infected livestock due to one infected Aedes spp. When climatic data are used, $\mathscr{R}_{0}$ is found to be more sensitive and elastic to the expected number of infected eggs laid by Aedes spp. via transovarial transmission, followed by the expected number of infected livestock due to one infected Aedes spp. and the expected number of infected Aedes spp. due to one infected livestock for both regions Arusha and Dodoma. These results call for attention to parameters regarding incubation period, the adequate contact rate of Aedes spp. and livestock, the infective periods of livestock and Aedes spp., and the vertical transmission in Aedes species.
\end{abstract}

\section{Introduction}

Rift valley fever (RVF) is a viral disease that primarily affects animals (such as sheep, horses, cattle, goats, camels, and buffalos) and has the capacity to affect human beings. Rift valley fever virus (RVFV) is a member of the Phlebovirus genus family Bunyaviridae which has been isolated from at least 40 mosquito species in the filed and other arthropods $[1,2]$. RVFV infection can cause severe disease in both animals and humans, leading to high disease induced death rate in livestock, long-term health effects in humans, and economic destruction of people $[3,4]$. Currently, two types of vaccine for animals exist: a live vaccine and inactivated vaccine. However, the current live vaccine cannot be used for prevention, and prevention using the inactivated vaccine is difficult to sustain in RVF affected countries for economic reasons $[3,5]$.

RVF can be transmitted through an initial aerosol release and subsequent transmission through the mosquito vector. RVFV can remain dormant in Aedes spp. mosquito eggs in dry soil for years. During periods of heavy rainfall, larval habitats frequently become flooded, enabling the eggs to hatch and the mosquito population to rapidly increase, spreading the virus to animals on which they feed $[6,7]$. Among animals, RVFV is spread primarily by the bite of infected mosquitoes, mainly Aedes and Culex spp. which can acquire the virus from feeding on an infected animal [8-10]. The female Aedes spp. mosquito is also capable of transmitting the virus directly to her offspring (vertical transmission) via eggs leading to new generations of infected mosquitoes hatching from eggs $[11,12]$. This is not the case for Culex spp. mosquito.

RVFV can be transmitted to humans through the handling of animal tissue during slaughtering or butchering, assisting with animal births, conducting veterinary procedures, or from the disposal of carcasses or fetuses. Human infections have also resulted from the bites of infected mosquito vector, and by ingesting unpasteurized or uncooked milk and meat of infected animals [8-10, 13]. Transmission 
of RVFV by blood feeding flies is also possible. To date no human-to-human transmission of RVF has been documented [12].

RVF was first reported in Kenya (Africa) in 1931 [8], and it was primarily considered to be of sub-Saharan Africa until September, 2000, when RVF cases were confirmed in Saudi Arabia and Yemen (outside Africa) [14]. The recent outbreak in East Africa is that of 2006-2007 where 684 cases and 155 deaths were confirmed in Kenya, and 264 cases and 109 deaths in Tanzania. There were outbreaks also in Somalia and Sudan in the same period [12].

RVF outbreaks in East Africa have been largely correlated with the unusual heavy rainfall associated with El Nino [15], which consequently flooded many Aedes spp. breeding habitats. The hatching dynamics of Aedes spp. mosquitoes, the main reservoir of RVF in Africa, strongly depends on the rainfall pattern [1]. Eggs need to be flooded to hatch; thus, heavy rainfall results in a massive hatching episode and, consequently, the development of a large vector population. Once infection has been amplified in livestock, secondary vectors such as Culex spp. and other biting flies, which breed in semipermanent pools of water, become involved in the transmission of the virus [16].

Global temperature change, on the other hand, would affect the biology of the vectors, including feeding rate and egg production, and the length of the development cycle and the extrinsic incubation period. This may result in high vector density, an increased vector capacity to transmit the virus and a higher transmission rate [16]. When temperature rises above the biological maximum threshold for a species, it may decrease the vector population. Sustained climate shifts may lead to changes in the RVF burden in endemic areas and new outbreaks in areas of similar conditions. Thus, modeling the impact of climate change in the dynamics of RVF and its interventions is important for understanding of the disease.

Mathematical epidemiological models have been developed to assess the dynamics of RVF. Gaff et al. [17] proposed a theoretical model in a closed system which included two mosquito populations Aedes and Culex spp. and a livestock population. Their proposed model was a system of ordinary differential equations developed to explain the behaviour of the RVF transmission. The result of the development process was the production of a first-time model of this disease. The model was later modified by Gaff et al. [18] to assess the relative effectiveness of RVF countermeasures such as vector adulticide, vector larvicide, livestock vaccination, and livestock culling.

A theoretical model involving mosquito population, livestock and human population has been developed to study the dynamics of the disease using nonlinear differential equations [19]. The results show that the disease prevalence in both human and livestock is more sensitive to livestock and human recruitment rates suggesting isolation of livestock from human as a viable measure during the outbreak. The initial transmission and disease prevalence were found to be highly linked to mosquito population suggesting control measures such as vector adulticides and larvicides to be applied to reduce the mosquito population.
Fischer et al. [20] investigated the transmission potential of RVFV among livestock in the Netherlands. The model included the effect of temperature on the biting rate, mosquito population size, and the mortality of the vectors. The results show that high degree of vaccination and vector control strategy are needed to prevent RVF outbreaks. Other studies include that of Xue et al. [21] who developed a network-based metapopulation model approach to RVF epidemics to assess the disease spread in both time and space using network theory, Xue et al. [22] who investigate the spread of RVFV when introduced in United States, Chitnis et al. [23] who developed a model to assess the effect of vertical transmission in vector-borne disease with applications to RVF, and Niu et al. [24] who developed an epidemiological model of RVF with spatial dynamics to study the spatial effects.

In this paper, we propose a model that assesses the impact of climate change on the dynamics of RVF. The approach is based on the previous model of RVF transmission by Mpeshe et al. [19] and modifications have been made to incorporate vertical transmission and climate-driven parameters. To simplify the model, only temperature and precipitation are considered in this study. While Aedes spp. mosquito eggs are naturally infected by RVF virus via vertical transmission, this is not a case for Culex spp. mosquito and, therefore, we assume vertical transmission in our model only for Aedes species. To accommodate the impact of climate change we assert that temperature and precipitation can affect the laying and hatching of the eggs as well as the death rate, the effective contact rate, and the incubation period of the mosquitoes. When the epizootic is very high human can also be a source of infection for mosquitoes [25] and, therefore, also we assert in our model the human-to-mosquito transmission when the mosquitoes feed on an infected human.

\section{Materials and Methods}

2.1. Model Formulation. The model considers three populations: mosquitoes, livestock, and humans with diseasedependent death rate for livestock and humans. The mosquito population is subdivided into two: Aedes species and Culex species. Due to vertical transmission in Aedes spp., we include both infected and uninfected eggs in the model for determining the effect of vertical transmission in the initial transmission of RVF. The mode of transmission of RVF virus from vector to host, host to host, and host to vector is shown in the model flowchart shown by Figure 1. The egg population of Aedes spp. consists of uninfected eggs $\left(X_{a}\right)$ and infected eggs $\left(Y_{a}\right)$. The population for adult Aedes spp. consists of susceptible adults $\left(S_{a}\right)$, latently infected adults $\left(E_{a}\right)$, and infectious adults $\left(I_{a}\right)$. The egg population of Culex spp. consists of uninfected eggs $\left(X_{c}\right)$ only and the population for adult Culex spp. consists of susceptible adults $\left(S_{c}\right)$, latently infected adults $\left(E_{c}\right)$, and infectious adults $\left(I_{c}\right)$. The livestock population consists of susceptible livestock $\left(S_{l}\right)$, latently infected livestock $\left(E_{l}\right)$, infectious livestock $\left(I_{l}\right)$, and recovered livestock $\left(R_{l}\right)$. The human population consists of susceptible humans $\left(S_{h}\right)$, latently infected humans $\left(E_{h}\right)$, 


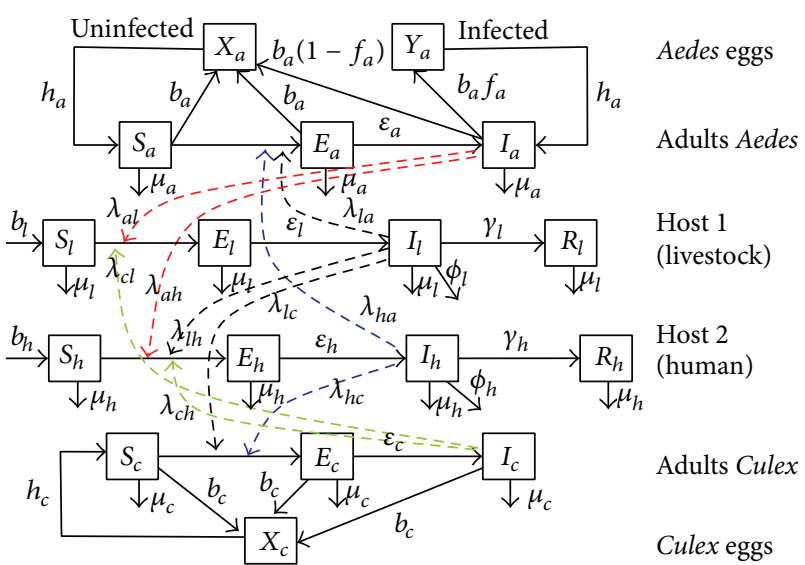

FIgURE 1: Flow diagram for the RVF model.

infectious humans $\left(I_{h}\right)$ and recovered humans $\left(R_{h}\right)$. Table 1 shows the model parameters and their description as they have been used in this work. $T$ and $P$ represent temperature and precipitation, respectively.

The epidemiology cycle of RVF presented by Balenghien et al. [26] and Chevalier et al. [27] is here applied to develop the flow diagram shown by Figure 1. The inclusion of the transmission dynamics of RVF from Aedes spp. to human and vice versa is due to the fact that some Aedes spp. such as Aedes vexans, Aedes aegpti, Aedes albopictus, Ae. ochraceus, Ae. mcintonshi, and Ae. dalzieli and many others numerously feed on humans, and therefore has the capacity to cause infection to human [26-31].

Using the parameters in Table 1 and the model flow diagram in Figure 1, an SEIR model is derived on the basis of the explanations above using first-order nonlinear ordinary differential equations as follows:

Aedes Mosquito

$$
\begin{gathered}
\frac{d X_{a}}{d t}=b_{a}(T, P)\left(N_{a}-f_{a} I_{a}\right)-h_{a}(T, P) X_{a}, \\
\frac{d Y_{a}}{d t}=b_{a}(T, P) f_{a} I_{a}-h_{a}(T, P) Y_{a}, \\
\frac{d S_{a}}{d t}=h_{a}(T, P) X_{a}-\mu_{a}(T) S_{a} \\
\quad-\lambda_{l a}(T) \frac{I_{l}}{N_{l}} S_{a}-\lambda_{h a}(T) \frac{I_{h}}{N_{h}} S_{a}, \\
\frac{d E_{a}}{d t}=\lambda_{l a}(T) \frac{I_{l}}{N_{l}} S_{a}+\lambda_{h a}(T) \frac{I_{h}}{N_{h}} S_{a}-\left(\varepsilon_{a}(T)+\mu_{a}(T)\right) E_{a}, \\
\frac{d I_{a}}{d t}=h_{a}(T, P) Y_{a}+\varepsilon_{a}(T) E_{a}-\mu_{a}(T) I_{a}, \\
\frac{d N_{a}}{d t}=
\end{gathered}
$$

Culex Mosquito

$$
\begin{gathered}
\frac{d X_{c}}{d t}=b_{c}(T, P) N_{c}-h_{c}(T, P) X_{c} \\
\frac{d S_{c}}{d t}=h_{c}(T, P) X_{c}-\mu_{c}(T) S_{c} \\
\quad-\lambda_{l c}(T) \frac{I_{1}}{N_{l}} S_{c}-\lambda_{h c}(T) \frac{I_{h}}{N_{h}} S_{c}, \\
\frac{d E_{c}}{d t}=\lambda_{l c}(T) \frac{I_{l}}{N_{l}} S_{c}+\lambda_{h c}(T) \frac{I_{h}}{N_{h}} S_{c}-\left(\varepsilon_{c}(T)+\mu_{c}(T)\right) E_{c}, \\
\frac{d I_{c}}{d t}=\varepsilon_{c}(T) E_{c}-\mu_{c}(T) I_{c}, \\
\frac{d N_{c}}{d t}=h_{c}(T, P) X_{c}-\mu_{c}(T) N_{c} .
\end{gathered}
$$

Livestock

$$
\begin{gathered}
\frac{d S_{l}}{d t}=b_{l} N_{l}-\mu_{l} S_{l}-\lambda_{a l}(T) \frac{I_{a}}{N_{a}} S_{l}-\lambda_{c l}(T) \frac{I_{c}}{N_{c}} S_{l}, \\
\frac{d E_{l}}{d t}=\lambda_{a l}(T) \frac{I_{a}}{N_{a}} S_{l}+\lambda_{c l}(T) \frac{I_{c}}{N_{c}} S_{l}-\left(\varepsilon_{l}+\mu_{l}\right) E_{l}, \\
\frac{d I_{l}}{d t}=\varepsilon_{l} E_{l}-\left(\mu_{l}+\phi_{l}+\gamma_{l}\right) I_{l}, \\
\frac{d R_{l}}{d t}=\gamma_{l} I_{l}-\mu_{l} R_{l}, \\
\frac{d N_{l}}{d t}=\left(b_{l}-\mu_{l}\right) N_{l}-\phi_{l} I_{l} .
\end{gathered}
$$

Humans

$$
\begin{aligned}
& \frac{d S_{h}}{d t}= b_{h} N_{h}-\mu_{h} S_{h}-\lambda_{l h} \frac{I_{l}}{N_{l}} S_{h} \\
&-\lambda_{a h}(T) \frac{I_{a}}{N_{a}} S_{h}-\lambda_{c h}(T) \frac{I_{c}}{N_{c}} S_{h}, \\
& \frac{d E_{h}}{d t}= \lambda_{l h} \frac{I_{l}}{N_{l}} S_{h}+\lambda_{a h}(T) \frac{I_{a}}{N_{a}} S_{h} \\
&+\lambda_{c h}(T) \frac{I_{c}}{N_{c}} S_{h}-\left(\varepsilon_{h}+\mu_{h}\right) E_{h}, \\
& \frac{d I_{h}}{d t}= \varepsilon_{h} E_{h}-\left(\mu_{h}+\phi_{h}+\gamma_{h}\right) I_{h}, \\
& \frac{d R_{h}}{d t}=\gamma_{h} I_{h}-\mu_{h} R_{h}, \\
& \frac{d N_{h}}{d t}=\left(b_{h}-\mu_{h}\right) N_{h}-\phi_{h} I_{h} .
\end{aligned}
$$

To test whether the model is well posed epidemiologically and mathematically, we need to investigate the feasibility of the model solution. Since $R_{l}$ and $R_{h}$ can be determined when $S_{l}, S_{h}, E_{l}, E_{h}, I_{l}$, and $I_{h}$ are known, without loss of generality, we omit the expression for $d R_{l} / d t$ and $d R_{h} / d t$ and write the system in compact form as

$$
\frac{d X}{d t}=M(x) X+F
$$


TABLE 1: Parameters used in the model formulation and their description.

\begin{tabular}{|c|c|c|}
\hline Parameter & Description & Dependent on climate change \\
\hline $1 / h_{a}(T, P)$ & Development time of Aedes mosquitoes & Temperature and precipitation \\
\hline $1 / h_{c}(T, P)$ & Development rate of Culex mosquitoes & Temperature and precipitation \\
\hline$b_{a}(T, P)$ & Number of Aedes eggs laid per day & Temperature and precipitation \\
\hline$b_{c}(T, P)$ & Number of Culex eggs laid per day & Temperature and precipitation \\
\hline$b_{h}$ & Daily birth rate in humans & Not considered \\
\hline$b_{l}$ & Daily birth rate in livestock & Not considered \\
\hline $1 / \mu_{a}(T)$ & Lifespan of Aedes mosquitoes & Temperature \\
\hline $1 / \mu_{c}(T)$ & Lifespan of Culex mosquitoes & Temperature \\
\hline $1 / \mu_{h}$ & Lifespan of humans & Not considered \\
\hline $1 / \mu_{l}$ & Lifespan of livestock & Not considered \\
\hline $1 / \varepsilon_{a}(T)$ & Incubation period of Aedes mosquitoes & Temperature \\
\hline $1 / \varepsilon_{c}(T)$ & Incubation period of Culex mosquitoes & Temperature \\
\hline $1 / \varepsilon_{h}$ & Incubation period of humans & Not considered \\
\hline $1 / \varepsilon_{l}$ & Incubation period of livestock & Not considered \\
\hline$\phi_{l}$ & Death rate of livestock due to disease & Not considered \\
\hline$\phi_{h}$ & Death rate of humans due to disease & Not considered \\
\hline $1 / \gamma_{l}$ & Infectious period in livestock & Not considered \\
\hline $1 / \gamma_{h}$ & Infectious period in humans & Not considered \\
\hline$\lambda_{\mathrm{al}}(T)$ & Adequate contact rate: Aedes to livestock & Temperature \\
\hline$\lambda_{\mathrm{cl}}(T)$ & Adequate contact rate: Culex to livestock & Temperature \\
\hline$\lambda_{\mathrm{la}}(T)$ & Adequate contact rate: livestock to Aedes & Temperature \\
\hline$\lambda_{\mathrm{lc}}(T)$ & Adequate contact rate: livestock to Culex & Temperature \\
\hline$\lambda_{\mathrm{ah}}(T)$ & Adequate contact rate: Aedes to humans & Temperature \\
\hline$\lambda_{\mathrm{ch}}(T)$ & Adequate contact rate: Culex to humans & Temperature \\
\hline$\lambda_{\mathrm{ha}}(T)$ & Adequate contact rate: humans to Aedes & Temperature \\
\hline$\lambda_{\mathrm{hc}}(T)$ & Adequate contact rate: humans to Culex & Temperature \\
\hline$\lambda_{\mathrm{lh}}$ & Adequate contact rate: livestock to humans & Not considered \\
\hline$f_{a}$ & Vertical transmission rate in Aedes & Not considered \\
\hline
\end{tabular}

where $X=\left(X_{a}, Y_{a}, S_{a}, E_{a}, I_{a}, X_{c}, S_{c}, E_{c}, I_{c}, S_{l}, E_{l}, I_{l}, S_{h}, E_{h}\right.$, and, therefore, $\left.I_{h}\right)^{T}, M(x)$ is a 15 by 15 matrix, and $F$ is a column matrix.

Substituting $I_{a}=N_{a}-\left(S_{a}+E_{a}\right)$ in $d X_{a} / d t$, we have

$\frac{d X_{a}}{d t}=b_{a}(T, P) N_{a}\left(1-f_{a}\right)+b_{a} f_{a}\left(S_{a}+E_{a}\right)-h_{a}(T, P) X_{a}$

$$
M(x)=\left[\begin{array}{ccc}
M_{1}(x) & 0 & 0 \\
0 & M_{2}(x) & 0 \\
0 & 0 & M_{3}(x)
\end{array}\right],
$$

(6) where

$$
\begin{aligned}
& M_{1}(x)=\left[\begin{array}{ccccc}
-h_{a}(T, P) & 0 & b_{a}(T, P) f_{a} & b_{a}(T, P) f_{a} & 0 \\
0 & -h_{a}(T, P) & 0 & 0 & b_{a}(T, P) f_{a} \\
h_{a}(T, P) & 0 & -\left(\mu_{a}+A\right) & 0 & 0 \\
0 & 0 & A & -\left(\varepsilon_{a}(T, P)+\mu_{a}(T, P)\right) & 0 \\
0 & h_{a}(T, P) & 0 & \varepsilon_{a}(T, P) & -\mu_{a}(T, P)
\end{array}\right] \\
& M_{2}(x)=\left[\begin{array}{cccc}
-h_{c}(T, P) & 0 & 0 & 0 \\
h_{c}(T, P) & -\left(\mu_{c}(T, P)+B\right) & 0 & 0 \\
0 & B & -\left(\varepsilon_{c}(T, P)+\mu_{c}(T, P)\right) & 0 \\
0 & 0 & \varepsilon_{c}(T, P) & -\mu_{c}(T, P)
\end{array}\right] \text {, } \\
& M_{3}(x)=\left[\begin{array}{cccccc}
-\left(\mu_{l}+C\right) & 0 & 0 & 0 & 0 & 0 \\
C & -\left(\varepsilon_{l}+\mu_{l}\right) & 0 & 0 & 0 & 0 \\
0 & \varepsilon_{l} & -\left(\mu_{l}+\phi_{l}+\gamma_{l}\right) & 0 & 0 & 0 \\
0 & 0 & 0 & -\left(\mu_{h}+D\right) & 0 & 0 \\
0 & 0 & 0 & D & -\left(\varepsilon_{h}+\mu_{h}\right) & 0 \\
0 & 0 & 0 & 0 & \varepsilon_{h} & -\left(\mu_{h}+\phi_{h}+\gamma_{h}\right)
\end{array}\right]
\end{aligned}
$$


with

$$
\begin{gathered}
A=\lambda_{l a}(T) \frac{I_{l}}{N_{l}}+\lambda_{h a}(T) \frac{I_{h}}{N_{h},} \\
B=\lambda_{l c}(T) \frac{I_{l}}{N_{l}}+\lambda_{h c}(T) \frac{I_{h}}{N_{h}}, \\
C=\lambda_{a l}(T) \frac{I_{a}}{N_{a}}+\lambda_{c l}(T) \frac{I_{c}}{N_{c}}, \\
D=\lambda_{l h}(T) \frac{I_{l}}{N_{l}}+\lambda_{a h}(T) \frac{I_{a}}{N_{a}}+\lambda_{c h}(T) \frac{I_{c}}{N_{c}}, \\
F=\left(b_{a}(T, P) N a\left(1-f_{a}\right), 0,0,0,0, b_{c}(T, P) N_{c},\right. \\
\left.0,0,0, b_{l} N_{l}, 0,0, b_{h} N_{h}, 0,0\right)^{T} \geq 0 .
\end{gathered}
$$

Combining all together, the matrix $M(x)$ is a Metzler matrix for all $\mathbb{R}_{+}^{15}$. Therefore, the model system is positively invariant in $\mathbb{R}_{+}^{15}$, and $F$ is Lipschitz continuous. Thus, the feasible region for the model system is the set

$$
\begin{aligned}
\mathscr{D}=\{( & \left.X_{a}, Y_{a}, S_{a}, E_{a}, I_{a}, X_{c}, S_{c}, E_{c}, I_{c}, S_{l}, E_{l}, I_{l}, S_{h}, E_{h}, I_{h}\right) \\
& \left.\geq 0 \in \mathbb{R}_{+}^{15}\right\} .
\end{aligned}
$$

That is, the solution remains in the feasible region $\mathscr{D}$ if it starts in this region. Hence, it is sufficient to study the dynamics of the model in $\mathscr{D}$.

2.2. Climate Driven Parameters. Several parameters related to mosquito vector, such as the hatching rate, vector mortality and longevity, biting rate, and extrinsic incubation period, depend on the temperature and precipitation. Using the existing studies and information from Aedes vexans, Aedes aegypti, Culex pipiens, and Culex quinquefasciatus [20, 3234] which are potential vectors of RVF, we generalise the following relations for Aedes and Culex spp. mosquitoes.

2.2.1. Hatching Rate or Mosquito Birth Rate, $h(T, P)$. This is the number of eggs hatching into adult mosquitoes at a certain period of time which we also refer to as the mosquito birth rate. It will depend on the the daily survival probability $\rho$ from eggs to adults and the duration $d$ it takes to develop from eggs to adults. The daily survival probability is assumed to depend independently on temperature, precipitation/rainfall, and prolonged period of desiccation. Thus,

$$
\rho(T, P, D)=\rho(T) \rho(P) \rho(D),
$$

where $\rho(T)$ is the daily survival probability of immaturity due to temperature effect $T ; \rho(P)$ is the daily survival probability of immaturity due to precipitation effect $P$; and $\rho(D)$ is the daily survival probability of immaturity due to desiccation effect $D$. The duration of maturation $d$ is assumed to depend on temperature. Therefore, the hatching rate is given by

$$
h(T, P, D)=\frac{\rho(T, P, D)}{d(T)} .
$$

2.2.2. Survival Probability due to Temperature Effect $\rho(T)$. The daily survival probability $\rho(T)$ is affected by the duration of maturation $d(T)$ in exponential form, that is,

$$
\rho(T)=\exp \left\{-\frac{1}{d(T)}\right\} .
$$

Fitting the data from [34] we obtain that $1 / d(T)=\alpha_{1} T^{2}+$ $\alpha_{2} T+\alpha_{3}$ for Culex spp. and $1 / d(T)=\alpha_{1} T^{3}+\alpha_{2} T^{2}+\alpha_{3} T+$ $\alpha_{4}$ for Aedes spp., where $\alpha_{1}=0.0095, \alpha_{2}=-0.4684, \alpha_{3}=$ 5.8343 for Culex spp. and $\alpha_{1}=-0.0025, \alpha_{2}=0.2069, \alpha_{3}=$ $-5.5285, \alpha_{4}=48.2951$ for Aedes spp.

2.2.3. Survival Probability due to Precipitation Effect $\rho(P)$. Precipitation or rainfall is important in creating breeding sites for mosquitoes and causing massive hatching. But excessive rainfall increases mortality of immature due to flushing effect. Since rainfall has two effects, that is, positive and negative effect, we use the idea from [35] and assume the daily survival probability of immaturity due to precipitation effect to be

$$
\rho(P)=\left(1-\exp \left\{-\beta_{1}\left(P-P_{1}\right)\right\}\right)\left(1-\exp \left\{-\beta_{2}\left(P_{2}-P\right)\right\}\right),
$$

where $\beta_{i}(i=1,2)$ are the sensitivity parameters; $P_{1}$ is the minimum amount of rainfall to support maturity; and $P_{2}$ is the maximum amount of rainfall which reduces their survival. For computational purposes we set $\rho(P)=0$ for $P<P_{1}$ and for $P>P_{2}$.

2.2.4. Survival Probability due to Desiccation Effect $\rho(D)$. Lack of precipitation affects the development of the immature. Following the approach by [32] we define the daily survival probability due desiccation as

$$
\rho\left(D_{t}\right)=\frac{\exp \left(-\omega D_{t}\right)}{c+\exp \left(-\omega D_{t}\right)}
$$

where $D$ depends on precipitation $P$ and is defined as

$$
D_{t}= \begin{cases}D_{t-1}+1 & P_{t} \leq P_{\text {th }} \\ 0 & \text { otherwise }\end{cases}
$$

where $P_{\text {th }}$ is the threshold precipitation; $D_{t}$ is the number of consecutive days up to time $t$ when the precipitation $P_{t}$ was below the threshold $P_{\mathrm{th}}$; $\omega$ is the sensitivity parameter; and $c$ is the constant that ensures that $\rho\left(D_{t}\right)$ is close to 1 at small values of $D_{t}$.

2.2.5. Daily Egg Laying Rate $b(T)$. The egg laying rate is assumed to depend on the moisture index. High moisture index correlates with high egg laying rate [33]. To model the daily egg laying rate we employ the equation derived by Gong et al. [33] that

$$
\begin{aligned}
b(T, P)= & \text { Baseline Egg rate } \\
& +\frac{E_{\max }}{1+\exp \left\{-\left(\text { Moisture Index }-E_{\text {mean }}\right) / E_{\text {var }}\right\}},
\end{aligned}
$$


where Baseline Egg rate is the baseline for fecundity, $E_{\max }$ is the maximum daily egg laying rate, $E_{\text {mean }}$ is the mean of daily egg laying rate, and $E_{\mathrm{var}}$ is the variance function.

To compute the moisture index, we apply Thornthwaite's moisture index [36] that

$$
\text { Moisture } \operatorname{Index}\left(I_{m}\right)=100\left(\frac{r}{E_{0}}-1\right) \text {, }
$$

where $r$ is the precipitation rate, and $E_{0}$ is the potential evapotranspiration. In absence of the potential evapotranspiration, Linacre's method [37] can be applied. That is,

$$
E_{0}=\frac{700 T_{m} /(100-A)+15\left(T-T_{d}\right)}{(80-T)} \text { mmday }^{-1}
$$

where, $T_{m}=T+0.00 h$ with $h$ being the elevation (metres), $T$ is the mean temperature, $A$ is the latitude (degrees), and $T_{d}$ is the mean dew-point.

2.2.6. Longevity of Mosquitoes $1 / \mu(T)$. Different studies show that the longevity of mature mosquitoes also depends on the temperature. To model the longevity, equations deduced by Fischer et al. [20] are applied. That is,

$$
\frac{1}{\mu(T)}=a_{0}-a_{1} T
$$

where $a_{0}=25.8, a_{1}=0.45$ for Aedes spp., and $a_{0}=69.1, a_{1}=$ 2.14 for Culex spp.

2.2.7. Extrinsic Incubation Period of Mosquitoes $1 / \varepsilon(T)$. Extrinsic incubation period is the time between a blood meal on an infections host and the first successful transmission from vector to host during another blood meal. We also adapt the expressions by Fischer et al. [20]. That is,

$$
\frac{1}{\varepsilon(T)}=\varepsilon_{\max }-\varepsilon_{\text {slope }} T
$$

where $\varepsilon_{\max }=18.9, \varepsilon_{\text {slope }}=0.30$ for Aedes spp., and $\varepsilon_{\max }=$ $11.3, \varepsilon_{\text {slope }}=0.30$ for Culex spp.

2.2.8. Adequate Contact Rate $\lambda(T)$. Adequate contact rate is contact which is sufficient for transmission of the infection from an infective to a susceptible. Thus, in this study

adequate contact rate

$$
=\text { biting rate } \times \text { probability of transmission. }
$$

The biting rate depends on temperature, and we assume a linear relationship as in Fischer et al. [20]. That is,

$$
a(T)=a_{\text {slope }}\left(T-T_{\min }\right),
$$

where $a_{\text {slope }}=0.0173, T_{\min }=9.60$ for all mosquito species. Assume that the probability of transmission is independent to temperature, we have

$$
\begin{array}{ll}
\lambda_{a l}(T)=a_{\text {slope }}\left(T-T_{\min }\right) \rho_{a l}, & \rho_{a l}=0.70, \\
\lambda_{c l}(T)=a_{\text {slope }}\left(T-T_{\min }\right) \rho_{c l}, & \rho_{c l}=0.78, \\
\lambda_{l a}(T)=a_{\text {slope }}\left(T-T_{\min }\right) \rho_{l a}, & \rho_{l a}=0.38, \\
\lambda_{l c}(T)=a_{\text {slope }}\left(T-T_{\min }\right) \rho_{l c}, & \rho_{l c}=0.22, \\
\lambda_{a h}(T)=a_{\text {slope }}\left(T-T_{\min }\right) \rho_{a h}, & \rho_{a h}=0.01, \\
\lambda_{h a}(T)=a_{\text {slope }}\left(T-T_{\min }\right) \rho_{h a}, & \rho_{h a}=0.05, \\
\lambda_{c h}(T)=a_{\text {slope }}\left(T-T_{\min }\right) \rho_{c h}, & \rho_{c h}=0.01, \\
\lambda_{h c}(T)=a_{\text {slope }}\left(T-T_{\min }\right) \rho_{h c}, & \rho_{h c}=0.015 .
\end{array}
$$

2.3. The Basic Reproduction Number. The basic reproduction number $\mathscr{R}_{0}$ is computed using the method of next generation matrix as outlined by [38]. Let $k_{i j}$ be the expected number of the new cases of type $i$ caused by one infected individual of type $j$, during the entire period of infectiousness. Define a matrix $K$ whose entries are $k_{i j}$, that is, $K=\left[k_{i j}\right]$. Then, $\mathscr{R}_{0}=$ $\rho(K)$, where $\rho(K)$ is spectral radius of $K$. For our model, we define four type-at-infection consisting of two vectors and two hosts, namely, Aedes spp. (type 1), Culex spp. (type 2), livestock (type 3), and humans (type 4). The resulting next generation matrix is

$$
K=\left[\begin{array}{llll}
k_{11} & k_{12} & k_{13} & k_{14} \\
k_{21} & k_{22} & k_{23} & k_{24} \\
k_{31} & k_{32} & k_{33} & k_{34} \\
k_{41} & k_{42} & k_{43} & k_{44}
\end{array}\right],
$$

where $k_{11}$ is the expected number of infected eggs laid by Aedes spp. via transovarial transmission, $k_{12}$ is the expected number of infected Aedes spp. due to one infected Culex, $k_{21}$ is the expected number of infected Culex spp. due to one infected Aedes spp., $k_{13}$ is the expected number of infected Aedes spp. due to one infected livestock, $k_{31}$ is the expected number of infected livestock due to one infected Aedes spp., $k_{14}$ is the expected number of infected Aedes spp. due to one infected human, $k_{41}$ is the expected number of infected humans due to one infected Aedes spp., $k_{22}$ is the expected number of infected eggs laid by Culex spp. via transovarial transmission, $k_{23}$ is the expected number of infected Culex spp. due to one infected livestock, $k_{32}$ is the expected number of infected livestock due to one infected Culex spp., $k_{24}$ is the expected number of infected Culex spp. due to one infected human, $k_{42}$ is the expected number of infected humans due to one infected Culex spp., $k_{33}$ is the expected number of infected livestock due to one infected livestock, $k_{34}$ is the expected number of infected livestock due to one infected human, $k_{43}$ is the expected number of infected humans due to one infected livestock, and and $k_{44}$ is the expected number of infected humans due to one infected human.

Since there is no vertical transmission in Culex spp., then $k_{22}=0$. The same applies for $k_{33}$ and $k_{44}$. Also Aedes spp. 
cannot infect Culex spp. and vice versa; therefore, $k_{12}=k_{21}=$ 0 . Humans cannot infect livestock, so $k_{34}=0$. Hence, we have

$$
K=\left[\begin{array}{cccc}
k_{11} & 0 & k_{13} & k_{14} \\
0 & 0 & k_{23} & k_{24} \\
k_{31} & k_{32} & 0 & 0 \\
k_{41} & k_{42} & k_{43} & 0
\end{array}\right] .
$$

The entry $k_{i j}$ depends on the probability that the individual of type $j$ survives the incubation, the adequate contact rate: individual type $j$ to individual type $i$, and the infective period of individual of type $j$. For example, $k_{13}$ will depend on the probability that livestock survives the incubation period, the adequate contact rate from livestock to Aedes spp., and the infective period of livestock. We therefore derive the $k_{i j}$ values as follows:

$$
\begin{aligned}
& k_{11}=\frac{b_{a}(T, P) f_{a}}{\mu_{a}}, \quad k_{13}=\left(\frac{\varepsilon_{l}}{\varepsilon_{l}+\mu_{l}}\right)\left(\frac{\lambda_{l a}(T)}{\mu_{l}+\phi_{l}+\gamma_{l}}\right), \\
& k_{14}=\left(\frac{\varepsilon_{h}}{\varepsilon_{h}+\mu_{h}}\right)\left(\frac{\lambda_{h a}(T)}{\mu_{h}+\phi_{h}+\gamma_{h}}\right) \text {, } \\
& k_{23}=\left(\frac{\varepsilon_{l}}{\varepsilon_{l}+\mu_{l}}\right)\left(\frac{\lambda_{l c}(T)}{\mu_{l}+\phi_{l}+\gamma_{l}}\right) \text {, } \\
& k_{24}=\left(\frac{\varepsilon_{h}}{\varepsilon_{h}+\mu_{h}}\right)\left(\frac{\lambda_{h c}(T)}{\mu_{h}+\phi_{h}+\gamma_{h}}\right) \text {, } \\
& k_{31}=\left(\frac{\varepsilon_{a}(T)}{\varepsilon_{a}(T)+\mu_{a}(T)}\right)\left(\frac{\lambda_{a l}(T)}{\mu_{a}(T)}\right), \\
& k_{32}=\left(\frac{\varepsilon_{c}(T)}{\varepsilon_{c}(T)+\mu_{c}(T)}\right)\left(\frac{\lambda_{c l}(T)}{\mu_{c}(T)}\right) \text {, } \\
& k_{41}=\left(\frac{\varepsilon_{a}(T)}{\varepsilon_{a}(T)+\mu_{a}(T)}\right)\left(\frac{\lambda_{a h}(T)}{\mu_{a}(T)}\right) \text {, } \\
& k_{42}=\left(\frac{\varepsilon_{c}(T)}{\varepsilon_{c}(T)+\mu_{c}(T)}\right)\left(\frac{\lambda_{c h}(T)}{\mu_{c}(T)}\right) \text {, } \\
& k_{43}=\left(\frac{\varepsilon_{l}}{\varepsilon_{l}+\mu_{l}}\right)\left(\frac{\lambda_{l h}(T)}{\mu_{l}+\phi_{l}+\gamma_{l}}\right) \text {. }
\end{aligned}
$$

2.4. Sensitivity and Elasticity Analyses of $\mathscr{R}_{0}$. Sensitivities quantify how $\mathscr{R}_{0}$ changes in response to the small shifts in the value of a parameter, while elasticities quantify the proportional change in $\mathscr{R}_{0}$ in response to the proportional change in a parameter. Both sensitivity and elasticity values can be used to judge which parameters are important to measure accurately and where variation in parameters will translate into variation in $\mathscr{R}_{0}$.

Caswell [39] developed a way to quantify sensitivity and elasticity of the growth rate $\lambda$ to changes in vital rates $a_{i j}$ where $a_{i j}$ are the entries of population matrix $A$. That is, the sensitivity of the growth rate $\lambda$ to changes in vital rates $a_{i j}$ is given by

$$
s_{i j}=\frac{\partial \lambda}{\partial a_{i j}}=\frac{v_{i} w_{j}}{\langle\mathbf{w}, \mathbf{v}\rangle},
$$

where $\mathbf{w}$ and $\mathbf{v}$ are the right and left eigenvectors, respectively, corresponding to the dominant eigenvalue $\lambda$ of the matrix $A$, and $\langle\mathbf{w}, \mathbf{v}\rangle$ is the dot product of $\mathbf{w}$ and $\mathbf{v}$. In case $a_{i j}$ is a function of other lower-level parameters, then, the chain rule can be applied to estimate the sensitivity of $\lambda$ to changes in any model parameter $p$ as

$$
s(p)=\frac{\partial \lambda}{\partial p}=\sum_{i j} \frac{\partial \lambda}{\partial a_{i j}} \frac{\partial a_{i j}}{\partial p} .
$$

The elasticity of the growth rate $\lambda$ to changes in vital rates $a_{i j}$, the entries of population matrix $A$, is given by

$$
e_{i j}=\frac{\partial \log \lambda}{\partial \log a_{i j}}=\frac{a_{i j}}{\lambda} \frac{\partial \lambda}{\partial a_{i j}} .
$$

For $a_{i j}$ a function of other lower-level parameters $p$, the elasticity is given by

$$
e(p)=\frac{p}{\lambda} \frac{\partial \lambda}{\partial p}=\frac{p}{\lambda} \sum_{i j} \frac{\partial \lambda}{\partial a_{i j}} \frac{\partial a_{i j}}{\partial p} .
$$

The theory of sensitivity analysis developed for the matrix models by Caswell [39] can be extended to the disease models to study the sensitivity and elasticity of $\mathscr{R}_{0}$ to the changes in the reproduction numbers $k_{i j}$ or the parameters defining them. Thus, the sensitivity $s_{i j}$ of a matrix element $k_{i j}$ is defined as the change in the eigenvalue $\left(\mathscr{R}_{0}\right)$ due to change in $k_{i j}$ given by

$$
s_{i j}=\frac{\partial \mathscr{R}_{0}}{\partial k_{i j}} .
$$

For individual parameter, the sensitivity $s(p)$ is given by

$$
s(p)=\sum_{i j} \frac{\partial \mathscr{R}_{0}}{\partial k_{i j}} \frac{\partial k_{i j}}{\partial p} .
$$

The elasticity $e_{i j}$ of a matrix element $k_{i j}$ is defined as

$$
e_{i j}=\frac{k_{i j}}{\mathscr{R}_{0}} \frac{\partial \mathscr{R}_{0}}{\partial k_{i j}} \text {. }
$$

For individual parameters $p$, the elasticity is given by

$$
e(p)=\frac{p}{\mathscr{R}_{0}} \sum_{i j} \frac{\partial \mathscr{R}_{0}}{\partial k_{i j}} \frac{\partial k_{i j}}{\partial p} .
$$

In order to study the impact of climate change to climatedriven parameter in the distribution of $\mathscr{R}_{0}$ we use climate data from two different regions in Tanzania, namely, Arusha and Dodoma for the 2006-2007 outbreak. According to WHO [12], RVF was reported in 10 out of the 21 regions of Tanzania where 12 cases were reported in Arusha region, 1 in Dar es Salaam, 156 in Dodoma, 4 in Iringa, 6 in Manyara, 50 in Morogoro, 5 in Mwanza, 5 in the Pwani, 24 in Singida, and 1 in Tanga regions. From the data we find that Dodoma has more than $50 \%$ of the total cases giving a justification for being a case of study, and Arusha is considered in this study because the first case was reported in January 2007 in this region. 
TABLE 2: Parameters with their estimated lower and higher values without considering impact of climate change.

\begin{tabular}{lccc}
\hline Parameter & low value & high value & Reference \\
\hline $1 / b_{a}$ & 100 & 200 & Assumed \\
$1 / \mu_{a}$ & 3 days & 60 days & {$[17]$} \\
$1 / \mu_{c}$ & 3 days & 60 days & {$[17]$} \\
$1 / \mu_{h}$ & 40 yrs & 60 yrs & {$[19]$} \\
$1 / \mu_{l}$ & 1 yr & 10 yrs & {$[40]$} \\
$1 / \varepsilon_{a}$ & 4 days & 8 days & {$[19]$} \\
$1 / \varepsilon_{c}$ & 4 days & 8 days & {$[19]$} \\
$1 / \varepsilon_{h}$ & 2 day & 6 days & {$[19]$} \\
$1 / \varepsilon_{l}$ & 1 day & 6 days & {$[19]$} \\
$\phi_{l}$ & 0.025 & 0.10 & {$[19]$} \\
$\phi_{h}$ & 0.01 & 0.10 & {$[19]$} \\
$f_{a}$ & 0.05 & 0.1 & {$[18]$} \\
$1 / \gamma_{l}$ & 1 day & 5 days & {$[19]$} \\
$1 / \gamma_{h}$ & 4 days & 7 days & {$[19]$} \\
$\lambda_{a l}$ & 0.15 & 0.48 & {$[18]$} \\
$\lambda_{c l}$ & 0.05 & 0.13 & {$[18]$} \\
$\lambda_{l a}$ & 0.15 & 0.395 & {$[18]$} \\
$\lambda_{l c}$ & 0.15 & 0.56 & {$[18]$} \\
$\lambda_{a h}$ & 0.001 & 0.002 & {$[22]$} \\
$\lambda_{c h}$ & 0.0005 & 0.001 & {$[22]$} \\
$\lambda_{h a}$ & 0.001 & 0.0015 & Assumed \\
$\lambda_{h c}$ & 0.0015 & 0.002 & Assumed \\
$\lambda_{l h}$ & 0.001 & 0.002 & {$[19]$} \\
\hline & & &
\end{tabular}

\section{Results and Discussion}

In this section we first present the result for $\mathscr{R}_{0}$ when the parameters are assumed to be independent of climate change. Then, we will compute the numerical value for $\mathscr{R}_{0}$ when climate change is considered to climate-driven parameters. Sensitivity and elasticity analysis results in both cases will be presented. Table 2 shows the parameter values for low range and high range which are used to compute the numerical value for $\mathscr{R}_{0}$ when temperature and precipitation effects are not considered.

When we substitute the values in Table 2 to the expressions of the elements of matrix $K$ and compute $\mathscr{R}_{0}$, we obtain that for low parameter values $\mathscr{R}_{0}=0.1941$ and for high parameter values $\mathscr{R}_{0}=6.8071$.

When climate change parameters were evaluated using the climate variable the value of $\mathscr{R}_{0}$ change from 0.4747 to 14.2007 in Arusha with the highest value marked in November $2006(=14.2007)$ followed by December 2006 (= 14.1530). The value of $\mathscr{R}_{0}$ dropped below 1 in January 2007 and February 2007, but it rose again in March, April, and May. Figure 2 (a) shows the distribution of $\mathscr{R}_{0}$ from July 2006 to June 2007 in Arusha region.

In Dodoma, the highest $\mathscr{R}_{0}$ was marked February 2007 $(=12.7438)$ followed by January $2007(=12.7368)$ then March $2007(=7.9899)$ and December $2006(=1.5088)$ as Figure 2(b) indicates.
TABLE 3: Sensitivity and elasticity of $\mathscr{R}_{0}$ for low and high parameter values.

\begin{tabular}{lcc}
\hline Parameter & Sensitivity & Elasticity \\
\hline$k_{11}$ & Low parameter values \\
$k_{13}$ & 0.37750 & 0.00292 \\
$k_{14}$ & 0.49913 & 0.37438 \\
$k_{23}$ & 0.00629 & 0.00012 \\
$k_{24}$ & 0.16513 & 0.12386 \\
$k_{31}$ & 0.00208 & 0.00006 \\
$k_{32}$ & 0.37679 & 0.37443 \\
$k_{41}$ & 0.37399 & 0.12388 \\
$k_{42}$ & 0.01118 & 0.00007 \\
$k_{43}$ & 0.01109 & 0.00003 \\
& 0.01497 & 0.00008 \\
$k_{11}$ & High parameter values & \\
$k_{13}$ & 0.36274 & 0.00160 \\
$k_{14}$ & 1.87148 & 0.36107 \\
$k_{23}$ & 0.01149 & 0.00001 \\
$k_{24}$ & 0.50469 & 0.13804 \\
$k_{31}$ & 0.00310 & 0.00000 \\
$k_{32}$ & 0.09672 & 0.36107 \\
$k_{41}$ & 0.13653 & 0.13804 \\
$k_{42}$ & 0.00045 & 0.00001 \\
$k_{43}$ & 0.00063 & 0.00000 \\
& 0.00231 & 0.00000 \\
\hline
\end{tabular}

TABle 4: Sensitivity and elasticity of $\mathscr{R}_{0}$ for Dodoma and Arusha climate data.

\begin{tabular}{lcc}
\hline Parameter & Sensitivity & Elasticity \\
\hline$k_{11}$ & Dodoma & \\
$k_{13}$ & 0.99874 & 0.99748 \\
$k_{14}$ & 0.12996 & 0.00126 \\
$k_{23}$ & 0.00020 & 0.00000 \\
$k_{24}$ & 0.00010 & 0.00000 \\
$k_{31}$ & 0.00000 & 0.00000 \\
$k_{32}$ & 0.00971 & 0.00126 \\
$k_{41}$ & 0.00001 & 0.00000 \\
$k_{42}$ & 0.00010 & 0.00000 \\
$k_{43}$ & 0.00000 & 0.00000 \\
& 0.00001 & 0.00000 \\
$k_{11}$ & Arusha & \\
$k_{13}$ & 0.99921 & 0.99841 \\
$k_{14}$ & 0.10903 & 0.00079 \\
$k_{23}$ & 0.00016 & 0.00000 \\
$k_{24}$ & 0.00012 & 0.00000 \\
$k_{31}$ & 0.00000 & 0.00000 \\
$k_{32}$ & 0.00727 & 0.00079 \\
$k_{41}$ & 0.00000 & 0.00000 \\
$k_{42}$ & 0.00008 & 0.00000 \\
$k_{43}$ & 0.00000 & 0.00000 \\
& 0.00001 & 0.00000 \\
\hline
\end{tabular}




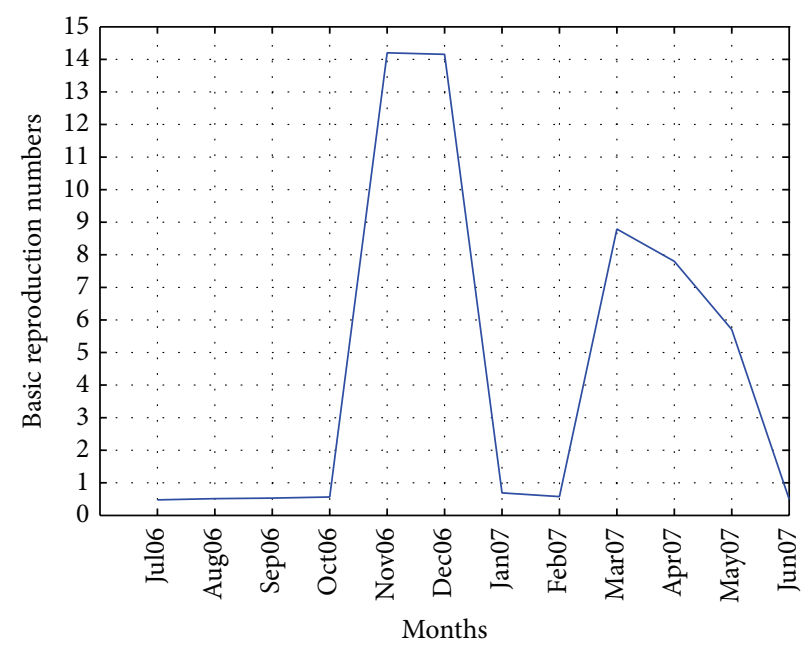

(a) Distribution of $\mathscr{R}_{0}$ for Arusha

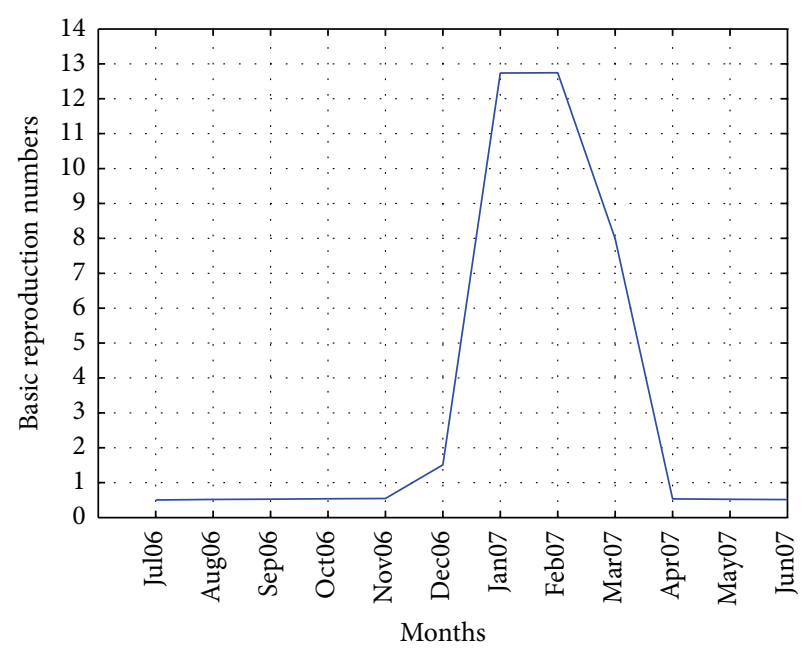

(b) Distribution of $\mathscr{R}_{0}$ for Dodoma

FIgURE 2: Distribution of $\mathscr{R}_{0}$ for climatic data in Arusha and Dodoma.

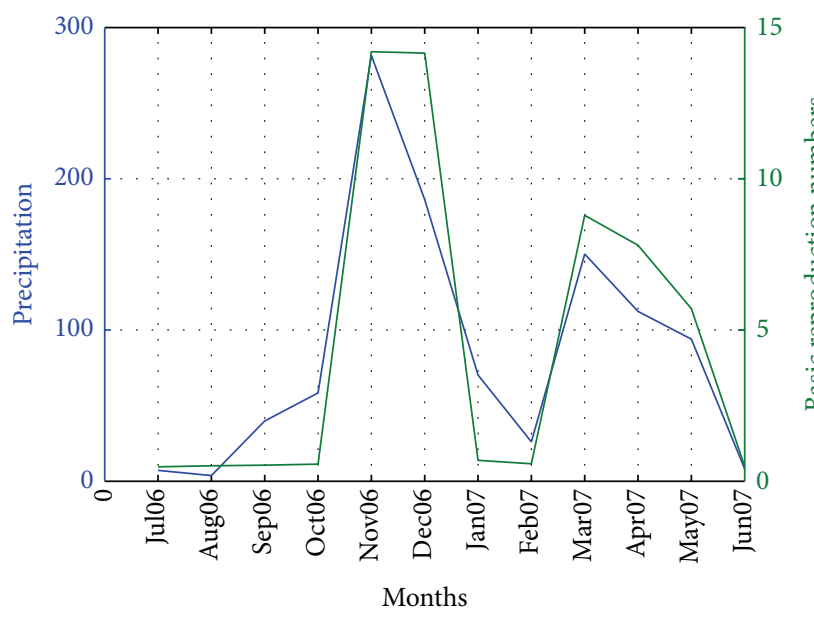

(a) $\mathscr{R}_{0}$ and precipitation for Arusha

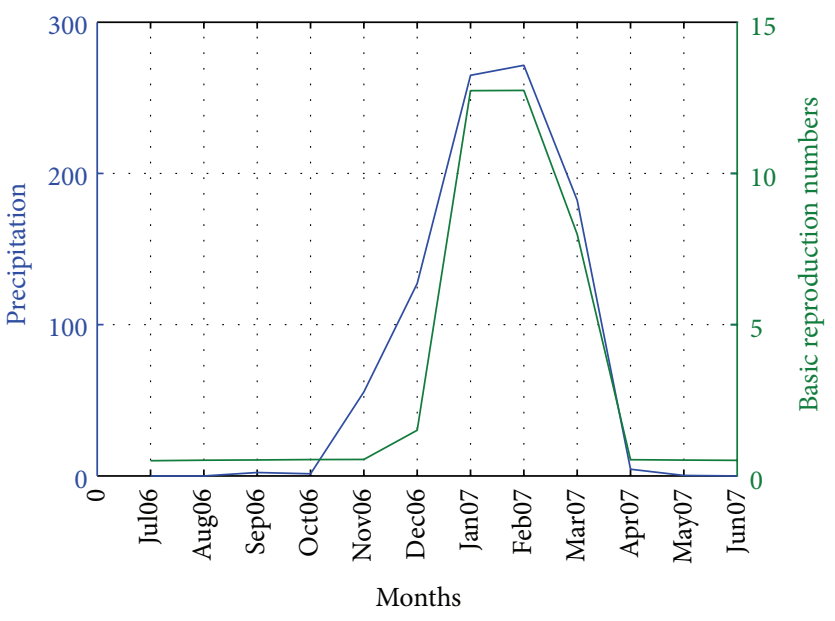

(b) $\mathscr{R}_{0}$ and precipitation for Dodoma

Figure 3: $\mathscr{R}_{0}$ and precipitation for climatic data in Arusha and Dodoma.

While it is clear that $\mathscr{R}_{0}$ increases with increase in rainfall, it is not the case for temperature where we experience high $\mathscr{R}_{0}$ for low temperatures. Figure 3 shows the plots for $\mathscr{R}_{0}$ and precipitation over months, and Figure 4 shows the plots for $\mathscr{R}_{0}$ and temperature over months.

Table 3 shows the sensitivity and elasticity values of $\mathscr{R}_{0}$, to both low and high parameter values. For both low and high parameter values, $\mathscr{R}_{0}$ is most sensitive to $k_{13}$, the expected number of infected Aedes spp. Due to one infected livestock, and to and most elastic to $k_{31}$, the expected number of infected livestock due to one infected Aedes spp. Table 3 shows the sensitivity and elasticity values of $\mathscr{R}_{0}$ for low and high parameter values, and Figure 5 shows the plots of sensitivity and elasticity values plotted against the parameter $k_{i j}$. The results suggest that attention should be given to parameters regarding incubation period, the adequate contact rate, and the infective period of livestock and Aedes spp.
When climatic data are used, $\mathscr{R}_{0}$ is found to be more sensitive and elastic to $k_{11}$, the expected number of infected eggs laid by Aedes spp. via transovarial transmission, followed by $k_{13}$ and $k_{31}$ for both regions Arusha and Dodoma. Table 4 shows the sensitivity and elasticity values of $\mathscr{R}_{0}$ for Dodoma and Arusha climate data, and Figure 6 shows the plots of sensitivity and elasticity against the parameters $k_{i j}$. The results call for attention to parameters regarding incubation period, the adequate contact rate of Aedes spp. and livestock, the infective periods of livestock and Aedes spp., and the vertical transmission in Aedes spp.

\section{Conclusion}

A deterministic SEIR model of RVF has been presented to study the impact of climate change variables mainly temperature and precipitation. The model presented here is just 


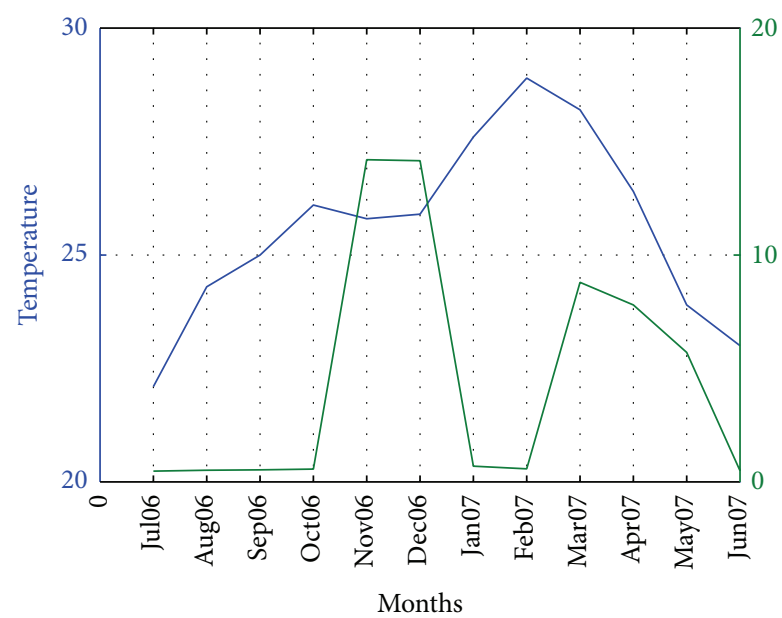

(a) $\mathscr{R}_{0}$ and temperature for Arusha
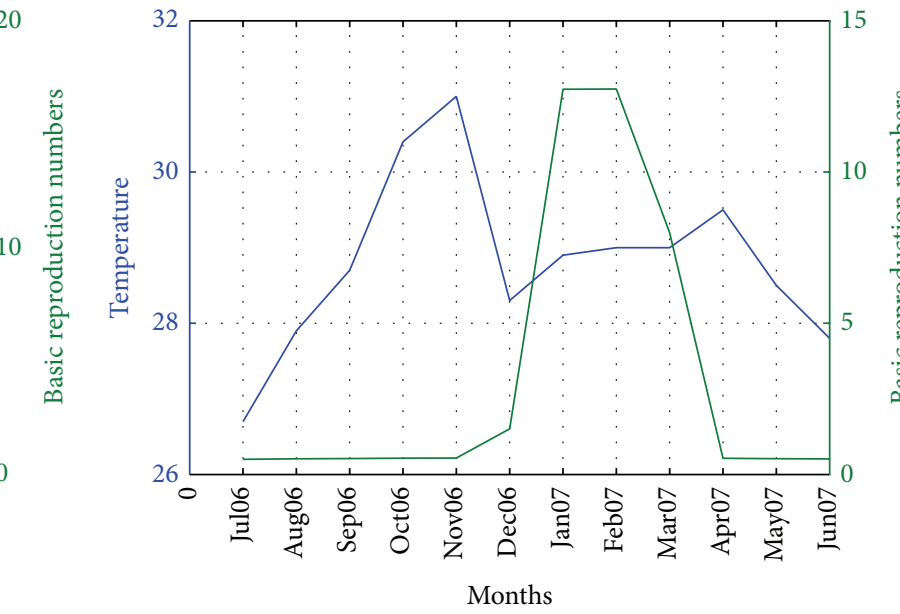

(b) $\mathscr{R}_{0}$ and temperature for Dodoma

FIgURE 4: $\mathscr{R}_{0}$ temperature for climatic data in Arusha and Dodoma.

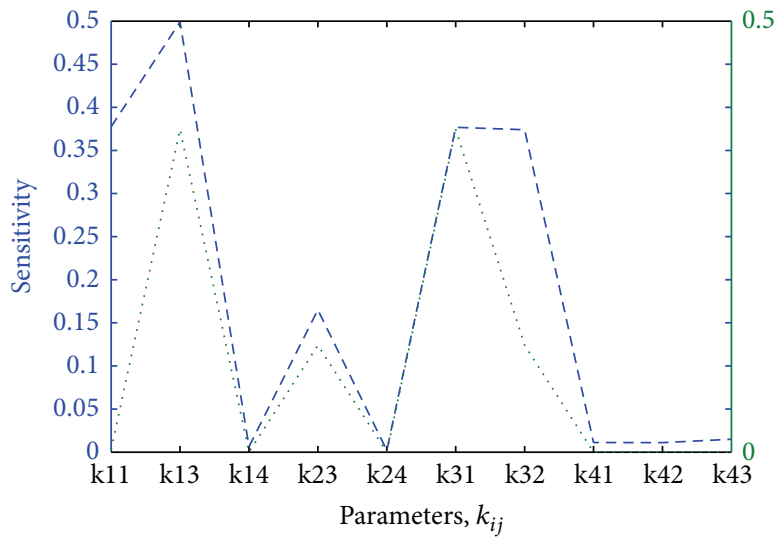

(a) Sensitivity and elasticity of $\mathscr{R}_{0}$ for low parameter values

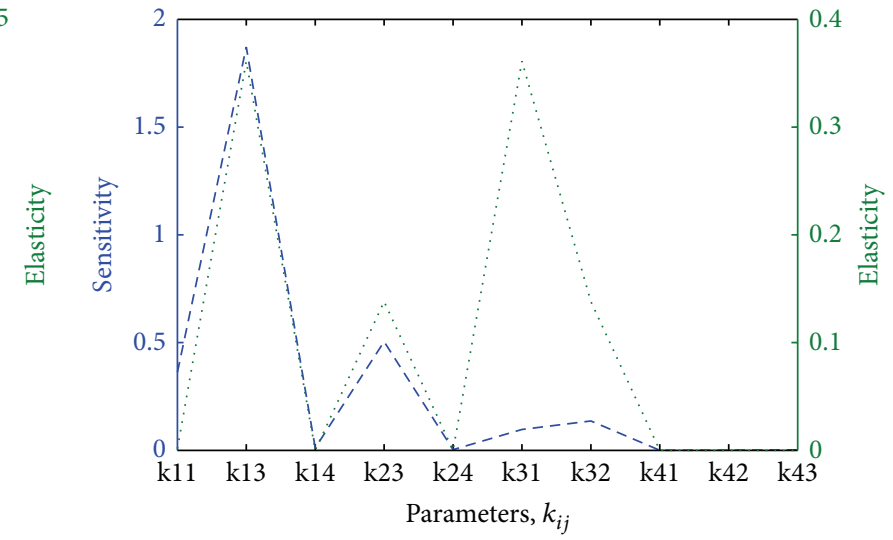

(b) Sensitivity and elasticity of $\mathscr{R}_{0}$ for high parameter values

FIGURE 5: Sensitivity and elasticity of $\mathscr{R}_{0}$ plotted against the low and high parameters values.

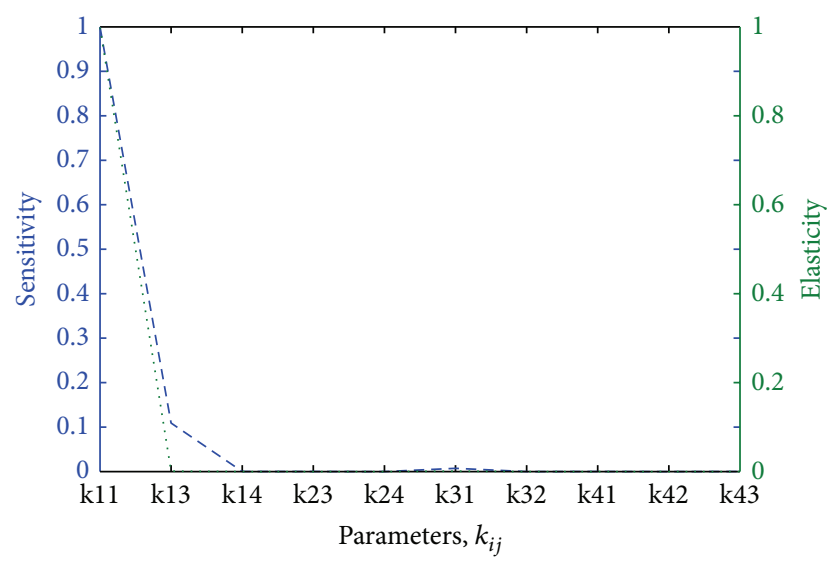

(a) Sensitivity and elasticity of $\mathscr{R}_{0}$ for Arusha

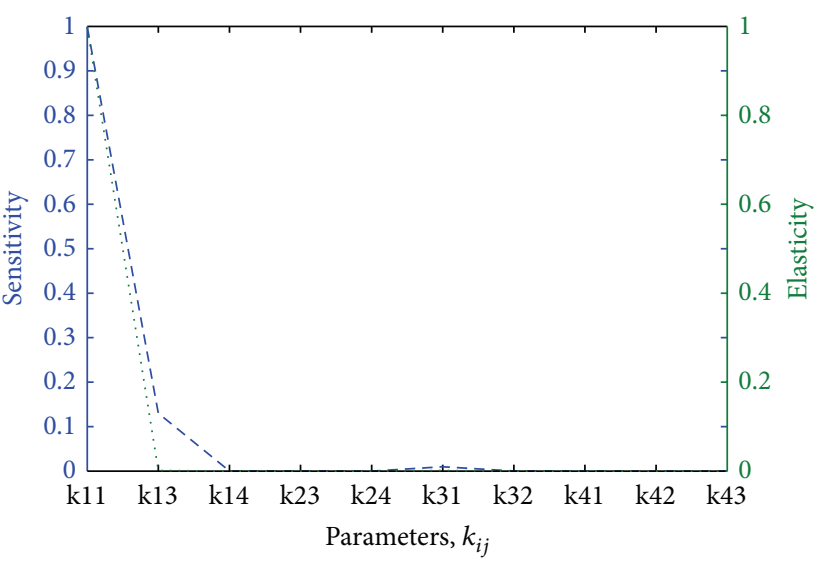

(b) Sensitivity and elasticity of $\mathscr{R}_{0}$ for Dodoma

FIgURE 6: Sensitivity and elasticity of $\mathscr{R}_{0}$ plotted against the parameters $k_{i j}$ for climatic data in Arusha and Dodoma. 
a simple representation of the complex ecological situation involved in the epidemiology of RVF. The formulation of the model, computation of $\mathscr{R}_{0}$, and sensitivity and elasticity analyses of $\mathscr{R}_{0}$ are based on the assumptions made to build the model as well as the chosen parameter values. Real climate data from Dodoma and Arusha where outbreak occured in 2006-2007 have been used to study the distribution of $\mathscr{R}_{0}$ in the whole period of the outbreak. Though the current analysis presented in this work may not be exhaustible, it remains, however, an important step toward the study of the impact of climate change on the dynamics of RVF.

\section{Conflict of Interests}

The authors declare that there is no conflict of interests regarding the publication of this paper.

\section{Acknowledgments}

The authors would like to thank the following institutions for support: Tanzania Meteorological Agency (TMA), University of Iringa, and Nelson Mandela African Institution of Science and Technology (NM-AIST). However, the conclusions made so far from this study are not influenced by any institution.

\section{References}

[1] J. M. Meegan and C. L. Bailey, "Rift Valley fever," in The Arboviruses: Epidemiology and Ecology, T. H. Monath, Ed., vol. 4, pp. 51-76, CRC Press, 1988.

[2] S. Moutailler, G. Krida, F. Schaffner, M. Vazeille, and A.-B. Failloux, "Potential vectors of rift valley fever virus in the Mediterranean region," Vector-Borne and Zoonotic Diseases, vol. 8, no. 6, pp. 749-753, 2008.

[3] FAO, "Rift Valley Fever: Vigilance Needed in the Coming Months," EMPRES WATCH, vol. 27, 2012.

[4] C. J. Peters and K. J. Linthicum, "Rift Valley fever," in Handbook of Zoonoses, G. B. Beran, Ed., pp. 125-138, CRC Press, Boca Raton, Fla, USA, 1994.

[5] M. Pepin, M. Bouloy, B. H. Bird, A. Kemp, and J. Paweska, "Rift Valley fever virus (Bunyaviridae: Phlebovirus): An update on pathogenesis, molecular epidemiology, vectors, diagnostics and prevention," Veterinary Research, vol. 41, no. 6, 2010.

[6] K. J. Linthicum, F. G. Davies, C. L. Bailey, and A. Kairo, "A mosquito species succession Dambo in an East African forest," Mosquito News, vol. 43, pp. 464-470, 1983.

[7] K. J. Linthicum, F. G. Davies, C. L. Bailey, and A. Kairo, "Mosquito species encountered in a flooded grassland Dambo in Kenya," Mosquito News, vol. 44, pp. 228-232, 1983.

[8] R. Daubney, J. R. Hudson, and P. C. Garnham, "Enzootic hepatitis of Rift Valley fever, an undescribed virus disease of sheep, cattle and man from East Africa," Journal of Pathology and Bacteriology, vol. 34, pp. 545-579, 1931.

[9] F. G. Davies, "Observations on the epidemiology of Rift Valley fever in Kenya," Journal of Hygiene, vol. 75, no. 2, pp. 219-230, 1975.

[10] G. R. Scott, W. Weddell, and D. Reid, "Preliminary finding on the prevalence of Rift Valley fever in Kenya Cattle," Bulletin of Epizootic Diseases of Africa, vol. 4, pp. 17-25, 1956.
[11] C. Favier, K. Chalvet-Monfray, P. Sabatier, R. Lancelot, D. Fontenille, and M. A. Dubois, "Rift Valley fever in West Africa: the role of space in endemicity," Tropical Medicine and International Health, vol. 11, no. 12, pp. 1878-1888, 2006.

[12] WHO, "Rift Valley fever," Fact Sheet 207, 2007, http://www .who.int/mediacentre/factsheets/fs207/en/.

[13] F. G. Davies and V. Martin, "Recognising Rift Valley fever," Veterinaria Italiana, vol. 42, no. 1, pp. 31-53, 2006.

[14] P. G. Jupp, A. Kemp, A. Grobbelaar et al., "The 2000 epidemic of Rift Valley fever in Saudi Arabia: Mosquito vector studies," Medical and Veterinary Entomology, vol. 16, no. 3, pp. 245-252, 2002.

[15] K. J. Linthicum, A. Anyamba, C. J. Tucker, P. W. Kelley, M. F. Myers, and C. J. Peters, "Climate and satellite indicators to forecast Rift Valley fever epidemics in Kenya," Science, vol. 285, no. 5426, pp. 397-400, 1999.

[16] V. Martini, V. Chevalier, P. Ceccato et al., "The impact of climate change on the epidemiology and control of Rift Valley fever," Revue Scientifique et Technique, vol. 27, no. 2, pp. 413-426, 2008.

[17] H. D. Gaff, D. M. Hartley, and N. P. Leahy, "An epidemiological model of rift valley fever," Electronic Journal of Differential Equations, vol. 115, pp. 1-12, 2007.

[18] H. Gaff, C. Burgese, J. Jackson, T. Niu, Y. Papelis, and D. Hartley, "Mathematical model to assess the relative effectiveness of Rift Valley fever countermeasures," International Journal of Artificial Life Research, vol. 2, no. 2, pp. 1-18, 2011.

[19] S. C. Mpeshe, H. Haario, and J. M. Tchuenche, "A mathematical model of Rift Valley fever with human host," Acta Biotheoretica, vol. 59, no. 3-4, pp. 231-250, 2011.

[20] E. A. J. Fischer, G. J. Boender, A. A. de Koeijer, H. A. Nodelijk, and H. J. van Roermund, "The transmission potential of Rift Valley fever virus among livestock in the Netherlands: a modelling study," Veterinary Research, vol. 44, article 58, 2013.

[21] L. Xue, H. H. Scott, and C. Scoglio, "A Network based Meta population approach to model Rift Valley epidemics," Journal of Theoretical Biology, vol. 306, pp. 129-144, 2012.

[22] L. Xue, H. H. Scott, L. W. Cohnstaedt, and C. Scoglio, "A hierarchical network approach for modeling Rift Valley fever epidemics," PLoS ONE, vol. 8, no. 5, Article ID e62049006204, 2013.

[23] N. Chitnis, J. M. Hyman, and C. A. Manore, "Modeling vertical transmision in vector-borne disease with applications to Rift Valley Fever," Journal of Biological Dynamics, vol. 7, pp. 11-40, 2013.

[24] N. Niu, H. D. Gaff, Y. E. Papelis, and D. M. Hartley, "An epidemiological model of Rift Valley fever with spatial dynamics," Computational and Mathematical Methods in Medicine, vol. 2012, Article ID 138757, 12 pages, 2012.

[25] A. R. Spickler, "Rift Valley Fever: infectious enzootic hepatitis of sheep and cattle," 2006, http://www.cfsph.iastate.edu/Factsheets/pdfs/rift_valley_fever.pdf.

[26] T. Balenghien, E. Cardinale, V. Chevalier et al., "Towards a better understanding of rift valley fever epidemiology in the South-West of the Indian Ocean," Vetenary Research, vol. 44, article 78, 2013.

[27] V. Chevalier, M. Pépin, L. Plée, and R. Lancelot, "Rift Valley fever: a threat for Europe?" Euro Surveillance, vol. 15, no. 10, Article ID 19506, 2010.

[28] J. D. Edman, "Fitness advantages in multiple blood-feeding: the Aedes aegypti example," in Ecological Aspects For Application of Genetically Modified Mosquitoes, W. Takken and T. W. Scott, Eds., vol. 2, 2003. 
[29] D. Fontenille, M. Traore-Lamizana, M. Diallo, J. Thonnon, J. P. Digoutte, and H. G. Zeller, "New vectors of Rift Valley fever in West Africa," Emerging Infectious Diseases, vol. 4, no. 2, pp. 289293, 1998.

[30] B. Kamgang, E. Nchoutpouen, F. Simard, and C. Paupy, "Notes on the blood-feeding behavior of Aedes albopictus (Diptera: Culicidae) in Cameroon," Parasites and Vectors, vol. 5, no. 1, article 57, 2012.

[31] A. Le Coupaner, D. Babin, L. Fiette et al., "Aedes mosquito saliva modulates Rift Valley fever pathogenicity," PLoS Neglected Tropical Diseases, vol. 7, no. 6, Article ID e2237, 2013.

[32] J. A. Ahumada, D. Lapointe, and M. D. Samuel, "Modeling the population dynamics of Culex quinquefasciatus (Diptera: Culicidae), along an elevational gradient in Hawaii," Journal of Medical Entomology, vol. 41, no. 6, pp. 1157-1170, 2004.

[33] H. Gong, A. De Gaetano, and L. Harrington, "A climate based mosquito population model," in Proceedings of the World Congress on Engineering and Computer Science, San Francisco, Calif, USA, 2007 October.

[34] L. M. Rueda, K. J. Patel, R. C. Axtell, and R. E. Stinner, "Temperature-dependent development and survival rates of Culex quinquefasciatus and Aedes aegypti (Diptera: Culicidae)," Journal of Medical Entomology, vol. 27, no. 5, pp. 892-898, 1990.

[35] P. Shi, F. Ge, Y. Sun, and C. Chen, "A simple model for describing the effect of temperature on insect developmental rate," Journal of Asia-Pacific Entemology, vol. 14, pp. 15-20, 2011.

[36] C. Thornthwaite and J. Mather, "The water balance," Meteorology, vol. 8, no. 1, pp. 1-104, 1955.

[37] E. T. Linacre, "A simple formula for estimating evaporation rates in various climates, using temperature data alone," Agricultural Meteorology, vol. 18, no. 6, pp. 409-424, 1977.

[38] C. Castillo-Chavez, Z. Feng, and W. Huang, "On the computation of $\mathscr{R}_{0}$ and its role in global stability," in Mathematical Approaches for Emerging and reemerging Infection Diseases: An Introduction, C. Castillo-Chavez, P. van den Driessche, D. Kirschner, and A. A. Yakubu, Eds., vol. 125, pp. 31-65, The IMA Volumes in Mathematics and its ApplicationsSpringer, New York, NY, USA, 2002.

[39] H. Caswell, Matrix Population Models: Construction, Analysis, and Interpretation, Sinauer Associates, Sunderland, Mass, USA, 2nd edition, 2001.

[40] O. Radostits, Herd Healthy: Food Animal Production Medicine, W.B. Saunders Company, Philidelphia, Pa, USA, 3rd edition, 2001. 


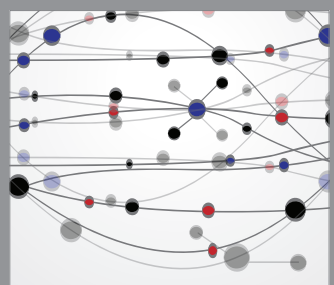

The Scientific World Journal
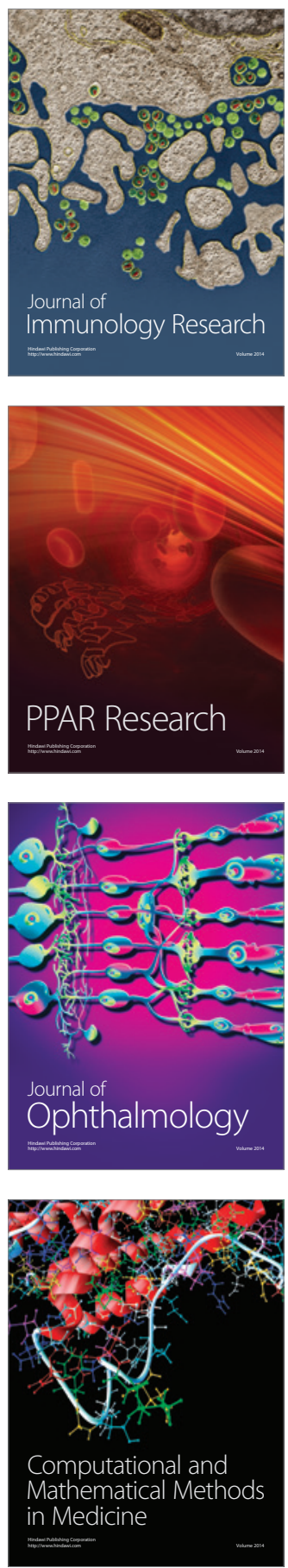

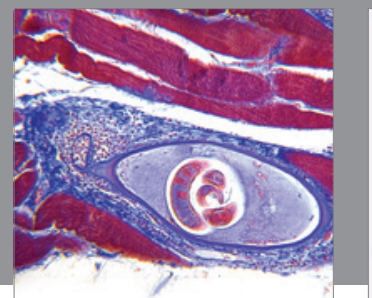

Gastroenterology

Research and Practice
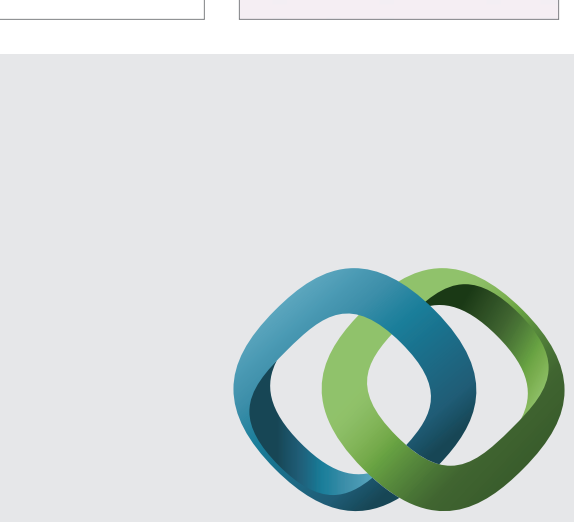

\section{Hindawi}

Submit your manuscripts at

http://www.hindawi.com
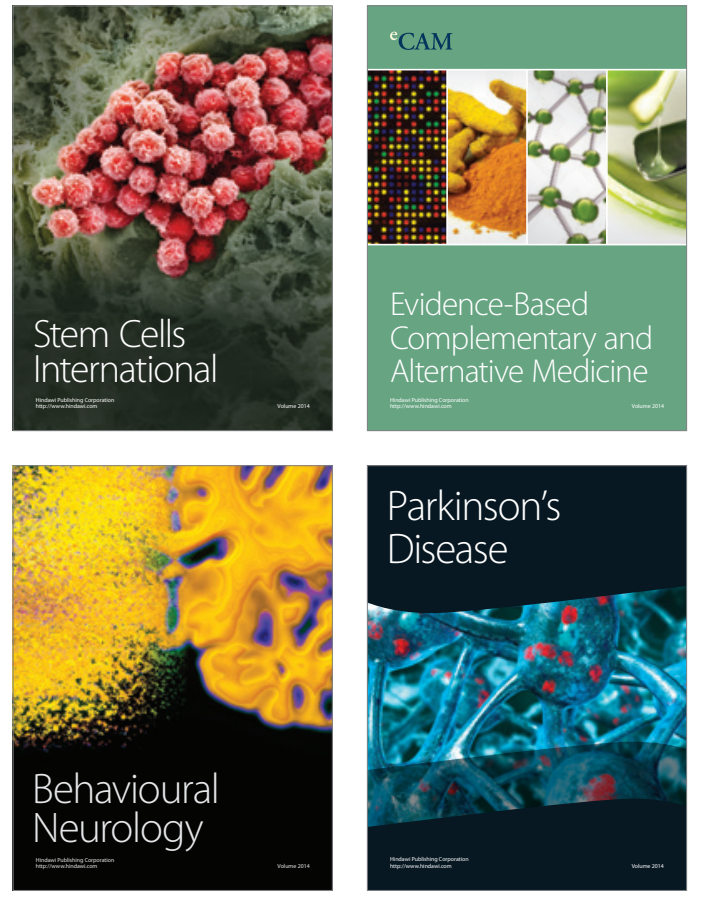
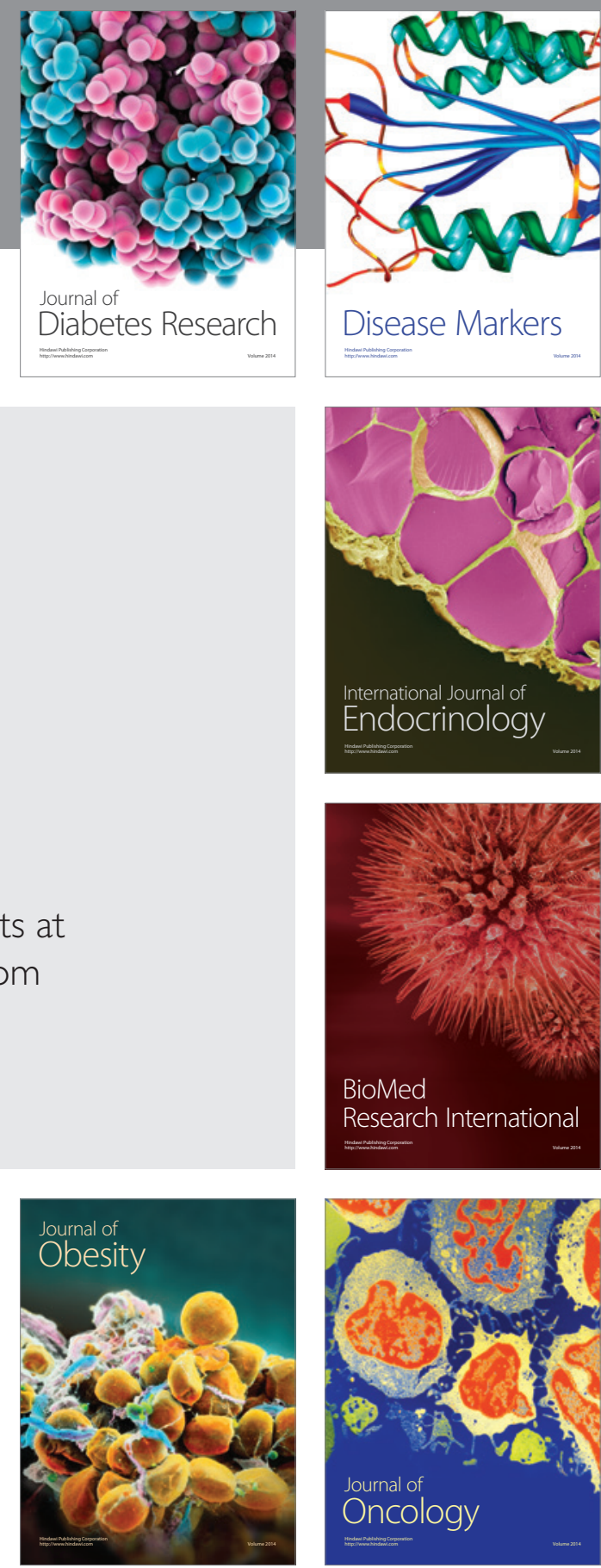

Disease Markers
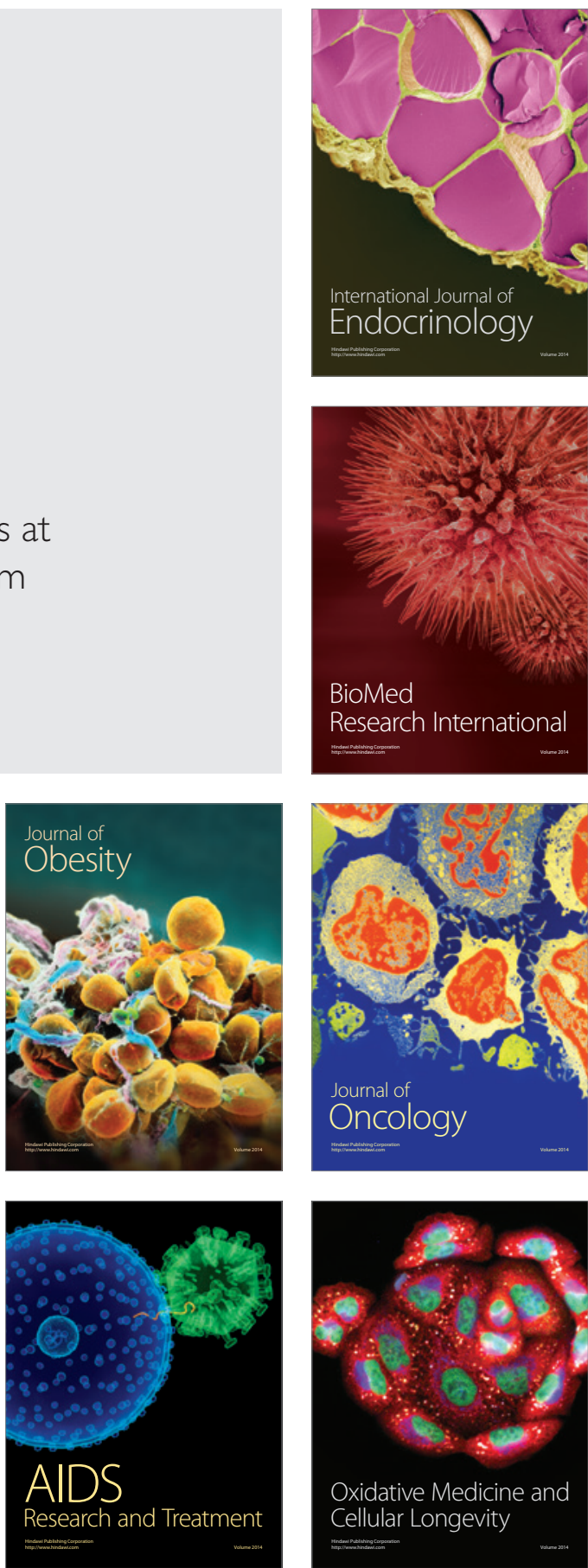